\section{LA EXPOSICIÓN A INFORMACIÓN POLÍTICA A TRAVÉS DE INTERNET}

\author{
Eva Anduiza \\ Camilo Cristancho \\ Universitat Autònoma de Barcelona \\ eva.anduiza@uab.cat \\ camilo.cristancho@gmail.com \\ Marta Cantijoch \\ University of Manchester \\ marta.cantijoch@manchester.ac.uk
}

\section{EXPOSURE TO POLITICAL INFORMATION ON THE INTERNET}

\begin{abstract}
This paper analyses online exposure to political information, its causes and some of its potential consequences. For this purpose we use survey data representative of the Spanish population gathered in 2007, 2008 and 2009. First we describe the extent to which Spanish citizens actively search for political information online or receive emails with political content. Secondly, we analyse which individual characteristics (socio-demographic variables, political attitudes and traditional media exposure) explain online exposure to political information. Thirdly, we study to what extent this variable affects political discussion and voter turnout in two election campaigns. Our findings show that only a very small portion of the population in Spain follows online news, and that socioeconomic resources and motivations are significant factors. The impact of this exposure is limited and has a bigger impact on political debate than voting.
\end{abstract}

KEY WORDS: turnout; political discussion; internet; online information; online campaign.

\section{INTRODUCCIÓN}

El uso de los nuevos medios por parte de candidatos, partidos y los electores en las campañas electorales ha generado grandes expectativas. Se espera que el uso de Internet en la comunicación política cambie las relaciones entre los ciudadanos y sus representantes, al mismo tiempo que surgen preguntas sobre las formas en que Internet puede incidir tanto positiva como negativamente sobre los valores del sistema democrático. Las preguntas relacionadas con las consecuencias del uso de Internet sobre el conocimiento político, las actitudes y los comportamientos de los ciudadanos buscan validar teorías que predicen efectos positivos del libre acceso, la personalización y la mayor oferta de información política en línea.
RESUMEN: En este artículo se analiza la exposición a información política a través de Internet, sus condicionantes y algunas de sus potenciales consecuencias. Para ello se utilizan datos procedentes de encuestas representativas de la población española recogidos en 2007, 2008 y 2009. En primer lugar se describe en qué medida los ciudadanos españoles buscan activamente información política online o reciben correos electrónicos con contenido político. En segundo lugar se analiza qué caracteristicas individuales (variables sociodemográficas, actitudes políticas y consumo de medios tradicionales) explican la exposición a información política online. En tercer lugar se estudian las consecuencias que ésta puede tener para la discusión política y la participación electoral en el contexto de dos campañas electorales. Los resultados permiten concluir que el seguimiento de la información política online es aún muy reducido en España y que ésta se ve condicionada tanto por los recursos socioeconómicos como por las motivaciones políticas. El efecto de esta exposición es limitado y más relevante para la discusión sobre política que para el voto.

PALABRAS CLAVE: participación electoral; discusión política; internet; información online; campaña online.

Para los más optimistas, la exposición a información política en línea puede favorecer la implicación de los ciudadanos en cuanto incrementan su conocimiento e interés por los asuntos públicos, la capacidad de adoptar una posición sobre los mismos, la percepción de que pueden incidir en ellos (eficacia política) y, consiguientemente, la participación. Diferentes investigaciones han encontrado pequeños efectos significativos de la exposición a la campaña online que sustentan ambas posiciones (Xenos y Moy, 2007; Kenski y Stroud, 2006; Cantijoch, Jorba y San Martin, 2008; Tolbert y McNeal, 2003 y Mossberger, Tolbert y McNeal, 2007). Para los más escépticos, estas potencialidades, si no se ven cumplidas, pueden generar frustración, y por lo tanto desafección. Argumentan que la web reflejará tan sólo factores políticos que ya están 
presentes en la sociedad (Margolis y Resnick, 2000) y que será por tanto una extensión de la vida política offline (Norris, 2003).

En este artículo se analizan tanto los condicionantes como las consecuencias del seguimiento de la información política online. Buscamos dar respuesta a preguntas como las siguientes: ¿Qué características tienen los ciudadanos que se informan online? ¿Son una parte significativa de la ciudadanía? ¿Con qué frecuencia y a través de qué medios específicos lo hacen? ¿Qué consecuencias tiene este seguimiento para su comportamiento? ¿Hablan más sobre política o sobre las elecciones? ¿Votan más?

Para ello nos centramos en tres momentos para los que tenemos disponibles datos de encuesta que permiten abordar estas preguntas. El estudio "Internet y participación política" del CIS, realizado en 2007 incluye abundante información sobre usos de Internet y participación política. Los estudios electorales de las elecciones generales de 2008 y de las europeas de 2009 también incluyen indicadores de consumo de información política online durante la campaña ${ }^{1}$. Estos tres momentos suponen diferentes escenarios tanto en términos de difusión de Internet en la sociedad (con usos mucho más diversificados en 2009), como en lo que se refiere a la intensidad de la movilización política (mucho mayor en elecciones generales). En la primera sección del artículo describimos la exposición a información política online en España. En la segunda parte nos centramos en los factores que explican este seguimiento. En un tercer apartado analizamos sus consecuencias respecto a la discusión política y al voto. Finalmente en las conclusiones discutimos el resultado de los análisis.

\section{LA EXPOSICIÓN A INFORMACIÓN POLÍTICA ONLINE EN ESPAÑA}

El acceso a información política online puede darse de tres maneras diferentes: los individuos buscan información política de manera deliberada (por ejemplo, a través de un buscador 0 accediendo a medios de comunicación online), pueden recibir contenidos por parte de terceras personas (por ejemplo, a través de un email), o bien pueden encontrarse con contenidos políticos de manera inadvertida mientras realizan otras actividades online (por ejemplo, navegando con otros propósitos).

La búsqueda de información política online se realiza con una intención previa que depende de un nivel mínimo de conocimiento y motivaciones hacia la política en general o la campaña electoral en concreto. Buscar información política en Internet exige una disposición más proactiva que en otros medios como la televisión, o incluso la prensa en papel, dadas las menores posibilidades de encontrar información específica sobre política dentro de una infinidad de fuentes y formatos online.

Recibir información con contenidos políticos puede darse como resultado de procesos previos de participación en campañas u otras instancias políticas no electorales, en los cuales los individuos solicitan información (se dan de alta en listas) generada por las campañas, organizaciones políticas o los medios, o bien mediante la recepción de mensajes que llegan sin previa solicitud por parte de amigos o conocidos. Las posibilidades de movilización política se han acrecentado de manera exponencial con la comunicación multicanal y las posibilidades de reutilización y personalización de la información que ofrece el uso de Internet. Por ello resulta de gran interés conocer qué tipo de personas reciben, generan y envían información política online. En efecto, la edad, la educación o los ingresos son variables que determinan la probabilidad de ser usuario de Internet, pero no tanto de hacerlo con fines políticos (Krueger, 2002 y Anduiza, Gallego y Cantijoch, 2010).

La exposición inadvertida a información política se da como producto de la casualidad de encontrar contenidos políticos mientras se utiliza Internet con otros fines. Tewksbury et al. (2001) analizaron las causas de encontrar noticias online mientras se realizaban otras actividades en Internet y concluyeron que las personas más jóvenes que pasan mayor tiempo online y que buscan información en general tienen mayor probabilidad de encontrar noticias. Esta exposición inadvertida ha sido mucho menos explorada, debido a que con frecuencia se ha asumido que Internet es un medio más eficiente que los tradicionales a la hora de ofrecer contenidos que se ajustan a las preferencias de los ciudadanos (Prior, 2007, pero véase Cantijoch, 2012 y Bode, 2010).

En España tenemos datos de encuesta sobre exposición a información política online general o relativa a la campa- 
ña electoral procedente de tres encuestas celebradas en momentos políticos muy diferentes. La comparación de los datos requiere comprender el contexto particular de cada encuesta y el enfoque de sus preguntas. La encuesta sobre "Internet y participación política" (2736) se realizó en otoño de 2007, más de tres años después de las últimas elecciones legislativas celebradas en marzo de 2004. Por esta razón las preguntas sobre búsqueda de información política y recepción de mensajes con contenido político son de alcance general ${ }^{2}$. Los estudios electorales del 2008 (2757) y 2009 (2807) incluyen preguntas sobre búsqueda de información y recepción de mensajes con contenido político específicamente relacionados con las campañas electorales generales y europeas ${ }^{3}$.
La tabla 1 resume los datos de exposición a información política online para las tres encuestas. Los datos no son estrictamente comparables dada la diferencia entre momentos políticos (electorales y no electorales), y en la intensidad de la campaña de las elecciones generales (2008) y las europeas (2009). Adicionalmente, debe considerarse que el número de internautas en España crece de forma estable en torno a los 500.000 nuevos usuarios cada 6 meses. Se estima que entre 2007 y 2008 el número de usuarios de Internet aumentó en 1,6 millones, considerando que el $60,5 \%$ de la población son usuarios habituales ${ }^{4}$. Debe notarse además el altísimo nivel de acceso a Internet entre jóvenes de 16 a 24 años con un $94,1 \% 0^{5}$.

TABLA 1. EXPOSICIÓN A INFORMACIÓN POLITICA ONLINE (PORCENTAJES SOBRE CONJUNTO DE LA MUESTRA)

\begin{tabular}{|c|c|c|c|}
\hline & $\begin{array}{c}2007 \\
N=3695\end{array}$ & $\begin{array}{c}2008 \\
N=6083\end{array}$ & $\begin{array}{c}2009 \\
N=3417\end{array}$ \\
\hline Ha buscado información sobre política/elecciones & $22 \%$ & $10 \%$ & $6,8 \%$ \\
\hline Ha recibido mensajes con contenido político/electoral ${ }^{a}$ & $21 \%$ & $8 \%$ & $3,5 \%$ \\
\hline Ha visto información política online sin buscarlab & ND & ND & $4,0 \%$ \\
\hline Lee información política en prensa ${ }^{c}$ & $68,9 \%$ & $54 \%$ & $46,4 \%$ \\
\hline
\end{tabular}

Fuente: Elaboración propia con datos de las encuestas CIS 2736 de 2007 y paneles electorales 2750, 2757 y 2800 y 2807.

a Para 2007, los mensajes incluyen apoyo a un partido, crítica a candidato, convocatoria manifestación, con una petición o manifiesto, con otros temas de actualidad.

${ }^{b}$ La pregunta para seguimiento de la campaña electoral en Internet mediante exposición a información recibida sin buscarla expresamente fue: "P32. ¿Ha recibido o accedido a información sobre la campaña o sobre política a través de Internet sin haberla buscado expresamente (a través del correo electrónico, Youtube, redes sociales como Facebook, etc.)?"

"Para 2008 y 2009 se refiere a información relativa a la campaña mientras que para 2007 se hace referencia a leer la prensa en general. Se toma el porcentaje de personas que lee la prensa con alguna frecuencia (frecuencia diferente de "nunca o casi nunca").

En 2007, el 22\% de los españoles afirmaron usar Internet para obtener información de actualidad política. Hay que tener en cuenta que este dato procede de un estudio monográfico sobre Internet y política, y por lo tanto es posible que la gente, sometida a varias preguntas sobre su actividad online, sea capaz de recordar mejor su contacto con información política online. En cambio en los estudios postelectorales hay muy pocas preguntas sobre usos de Internet y los indicadores se limitan a reflejar información sobre las elecciones (y no sobre política en general). Además en estos sondeos posteriores a una elección es habitual detectar un cierto efecto fatiga de los encuestados, sometidos a varias semanas de campaña electoral y saturación de información política. Posiblemente por todas estas razones los datos relativos a 2008 y 2009 son mucho más bajos. Sólo el 10\% del electorado buscó información sobre las elecciones durante la campaña de las generales de 2008. De éstos sólo el 20\% entró en webs de partidos o candidatos, y un porcentaje similar en blogs o foros de debate. Tan sólo el 3\% de los electores siguió la campaña del 2008 a diario a través de Internet frente a un porcentaje muy superior de quienes lo hicieron a través de la radio 
(20\%), la prensa (24\%) y la televisión (50\%). Para el 2009 las cifras son aún más bajas, posiblemente por cuenta de la menor resonancia mediática de las elecciones europeas. Menos del 7\% de los entrevistados buscó información sobre las elecciones y tan sólo el 3,5\% recibió mensajes con contenido electoral, frente a un $46 \%$ de la población que siguieron la campaña a través de la prensa.

La recepción de mensajes sobre las elecciones es muy inferior a la de mensajes sobre política en general (21\%). Además debe destacarse que para el caso de las elecciones europeas (2009) la recepción de mensajes con contenidos electorales fue menos de la mitad que para las elecciones generales (3,5 frente a $8 \%$, respectivamente). La exposición accidental a información política online solo se ha medido en 2009 y afecta a un $4 \%$ de la muestra $(8,1 \%$ de los usuarios de Internet). Esta cifra es muy baja en comparación con la que se ha podido medir en otros países como Estados Unidos 6 .

Estos resultados muestran una cierta variación en la medida y la forma en que Internet es utilizado para seguir las campañas electorales e información política en general. En todo caso los datos apuntan a que se trata de un medio aún muy minoritario y secundario con respecto a los tradicionales, y que España no se sitúa precisamente en los primeros puestos en seguimiento de la campaña online en el ámbito internacional'. Para elecciones generales, es difícil encontrar datos comparables y sistemáticos entre paises. En Francia el 44\% de los internautas declaraba en 2007 que había buscado información política online, el 26\% había visitado una web de algún candidato, el 19\% había visto vídeos políticos y el $18 \%$ había consultado blogs políticos (Vedel y Cann, 2008). En Noruega algo menos de la mitad de la población ha buscado información sobre la elección de 2005 en la web, aunque sólo el 13\% ha visitado la web de algún partido (Karlsen, 2007). Según el informe Pew ${ }^{8}$ sobre la elección presidencial de 2008, el 46\% de los norteamericanos utilizaron Internet, email o sms para informarse sobre la campaña, compartir sus puntos de vista o movilizar a otros. El 35\% mencionó haber mirado vídeos políticos online y el 10\% haber participado en redes sociales como Facebook o MySpace para conseguir información o implicarse.

Para las elecciones europeas de 2009 disponemos de la encuesta a votantes del European Election Studies de Piredeu, según el cual mientras que en 2004 tan sólo un
10\% de los europeos utilizó Internet como fuente de información sobre las elecciones europeas, para 2009 el uso de Internet como fuente de información política aumenta considerablemente. El rango oscila bastante y cambia considerablemente en cinco años desde un 8\% en Rumanía hasta 34\% en Suecia en comparación con 2004 cuando el uso de Internet como fuente de información oscilaba entre el 4\% en Grecia y el 14\% en Finlandia (Lusoli, 2005). España estaba próxima al resto de países del sur de Europa en torno al 6\% en 2004 y para 2009 está situada en el puesto 19 de 27 con un 15\%, cerca de países como Francia, Hungría, Estonia, Eslovaquia y Polonia (Tabla 2).

\section{¿QUIÉN BUSCA INFORMACIÓN POLITICA ONLINE?}

A continuación exploramos cuál es el perfil de las personas que en el caso español se informan online políticamente, ya sea porque buscan información activamente, o porque la reciben a través de emails. Buscamos dar respuesta a la pregunta ¿Qué características de los ciudadanos inciden en su probabilidad de exponerse a información política y electoral online? Esperamos un efecto significativo de las variables sociodemográficas (edad, género, estudios, ingresos), en la medida en que éstas indican el nivel de recursos de los individuos para emprender cualquier actividad política, tal como buscar información sobre política o sobre una campaña (Verba, Scholzman y Brady 1995). También esperamos que las actitudes políticas y el consumo de información política a través de medios tradicionales favorezcan esta exposición, en particular para la búsqueda de información, ya que ambos aspectos reflejan motivaciones políticas.

Las tablas 3 y 4 reflejan este análisis tanto para la población general (tabla 3) como para la población de jóvenes de hasta 30 años (tabla 4) ${ }^{9}$. Las variables dependientes son la búsqueda de información (política o electoral, según los años) y la recepción de emails (sobre política o sobre las elecciones). Como factores explicativos se han incluido el sexo, la edad, el nivel de estudios, los ingresos, el interés por la política, la ideología, la cercanía a un partido, las habilidades online, y el consumo de información política a través de medios tradicionales (prensa, televisión, radio). Detalles sobre la codificación de las variables pueden encontrarse en el apéndice. 
TABLA 2. BÚSOUEDA DE INFORMACIÓN POLITICA ONLINE SOBRE LAS ELECCIONES EUROPEAS

\begin{tabular}{|c|c|c|c|}
\hline & 2004 & 2009 & $\Delta \%$ \\
\hline Media & $10 \%$ & $20 \%$ & $11 \%$ \\
\hline Suecia & - & $34 \%$ & - \\
\hline Bélgica & $10 \%$ & $31 \%$ & $21 \%$ \\
\hline Finlandia & $19 \%$ & $31 \%$ & $12 \%$ \\
\hline Paises Bajos & $17 \%$ & $31 \%$ & $14 \%$ \\
\hline Dinamarca & $12 \%$ & $30 \%$ & $18 \%$ \\
\hline Letonia & $10 \%$ & $29 \%$ & $18 \%$ \\
\hline Luxemburgo & $7 \%$ & $26 \%$ & $19 \%$ \\
\hline Reino Unido & $10 \%$ & $25 \%$ & $15 \%$ \\
\hline Italia & $6 \%$ & $25 \%$ & $18 \%$ \\
\hline Austria & $15 \%$ & $24 \%$ & $8 \%$ \\
\hline Alemania & $12 \%$ & $22 \%$ & $11 \%$ \\
\hline Grecia & $12 \%$ & $22 \%$ & $10 \%$ \\
\hline Irlanda & $6 \%$ & $21 \%$ & $15 \%$ \\
\hline Eslovenia & $8 \%$ & $19 \%$ & $11 \%$ \\
\hline República Checa & $8 \%$ & $19 \%$ & $11 \%$ \\
\hline Malta & - & $18 \%$ & - \\
\hline Estonia & $10 \%$ & $16 \%$ & $6 \%$ \\
\hline Hungria & $6 \%$ & $16 \%$ & $10 \%$ \\
\hline España & $8 \%$ & $15 \%$ & $7 \%$ \\
\hline Francia & $8 \%$ & $15 \%$ & $7 \%$ \\
\hline Eslovaquia & $5 \%$ & $14 \%$ & $9 \%$ \\
\hline Polonia & $5 \%$ & $14 \%$ & $9 \%$ \\
\hline Chipre & $15 \%$ & $11 \%$ & $-4 \%$ \\
\hline Portugal & $10 \%$ & $11 \%$ & $2 \%$ \\
\hline Bulgaria & - & $10 \%$ & - \\
\hline Lituania & - & $9 \%$ & - \\
\hline Rumania & - & $8 \%$ & - \\
\hline Irlanda del Norte & $1 \%$ & - & - \\
\hline
\end{tabular}

Fuente: Voter Survey Data -de la encuesta "European Electoral Studies EES- Providing an Infrastructure for Research on Electoral Democracy in the European Union", del European University Institute y el Robert Schuman Centre for Advanced Studies. La pregunta cambió levemente de "iNavegó en las páginas web buscando información sobre las elecciones europeas?" en 2004 a "Exploró alguna web que trataba sobre las elecciones?". Datos acumulados para las respuestas "con frecuencia" y "algunas veces" N=1000 para cada país en 2004 (23 países) y N=1200 para 2009 (27 países). 
TABLA 3. ANÁLISIS DE LA BÚSOUEDA Y DE LA RECEPCIÓN DE INFORMACIÓN POLITICA ONLINE (REGRESIONES LOGISTICAS PARA TODA LA MUESTRA)

\begin{tabular}{|c|c|c|c|c|c|c|}
\hline & $\begin{array}{c}2007 \\
\text { Ha buscado } \\
\text { información } \\
\text { politica }\end{array}$ & $\begin{array}{c}2008 \\
\text { Ha buscado } \\
\text { información sobre las } \\
\text { elecciones generales }\end{array}$ & $\begin{array}{c}2009 \\
\text { Ha buscado } \\
\text { información sobre las } \\
\text { elecciones europeas }\end{array}$ & $\begin{array}{c}2007 \\
\text { Ha recibido un } \\
\text { email con contenido } \\
\text { politico }\end{array}$ & $\begin{array}{c}2008 \\
\text { Ha recibido un } \\
\text { email con contenido } \\
\text { político o electoral }\end{array}$ & $\begin{array}{c}2009 \\
\text { Ha recibido un } \\
\text { email con contenido } \\
\text { político o electoral }\end{array}$ \\
\hline \multirow{2}{*}{ Sexo (Mujer) } & $-0.384^{* *}$ & $-0.522^{* *}$ & -0.239 & 0.193 & -0.081 & 0.216 \\
\hline & $(0.121)$ & $(0.123)$ & $(0.176)$ & $(0.119)$ & $(0.127)$ & $(0.218)$ \\
\hline \multirow{2}{*}{ Edad } & -0.008 & $-0.043^{* *}$ & $-0.022^{* *}$ & $-0.017^{* *}$ & $-0.048^{* *}$ & $-0.024^{* *}$ \\
\hline & $(0.005)$ & $(0.005)$ & $(0.007)$ & $(0.005)$ & $(0.005)$ & $(0.009)$ \\
\hline \multirow{2}{*}{ Estudios } & $2.035^{* *}$ & $1.790^{* *}$ & $1.027^{*}$ & $1.630^{* *}$ & $2.269^{* *}$ & $2.650^{* *}$ \\
\hline & $(0.326)$ & $(0.281)$ & $(0.449)$ & $(0.324)$ & $(0.306)$ & $(0.621)$ \\
\hline \multirow{2}{*}{ Ingresos } & -0.526 & 0.504 & $1.098^{*}$ & $1.134^{* *}$ & $1.924^{* *}$ & $2.233^{* *}$ \\
\hline & (0.395) & $(0.346)$ & $(0.529)$ & $(0.386)$ & $(0.364)$ & $(0.667)$ \\
\hline \multirow{2}{*}{ Habilidades online ${ }^{a}$} & $4.777^{* *}$ & - & $0.427^{*}$ & $4.579^{* *}$ & - & $0.513^{*}$ \\
\hline & $(0.277)$ & - & $(0.179)$ & $(0.270)$ & - & $(0.233)$ \\
\hline \multirow{2}{*}{ Interés por la políticab } & $2.482^{* *}$ & $0.893^{* *}$ & $0.858^{*}$ & $1.512^{* *}$ & $1.429^{* *}$ & 0.319 \\
\hline & $(0.230)$ & $(0.227)$ & $(0.338)$ & $(0.220)$ & $(0.243)$ & $(0.430)$ \\
\hline \multirow{2}{*}{ Ideología (derecha) } & $-0.715^{*}$ & $-0.829^{* *}$ & 0.167 & -0.213 & -0.427 & -0.544 \\
\hline & $(0.294)$ & $(0.291)$ & $(0.393)$ & $(0.291)$ & $(0.301)$ & $(0.513)$ \\
\hline \multirow{2}{*}{ Partidismo } & $0.417^{* *}$ & 0.098 & $2.472^{* *}$ & $0.412^{* *}$ & $0.432^{* *}$ & $1.525^{* *}$ \\
\hline & $(0.134)$ & $(0.135)$ & $(0.290)$ & $(0.130)$ & $(0.147)$ & $(0.359)$ \\
\hline \multirow{2}{*}{ Lectura prensa } & $0.962^{* *}$ & $1.287^{* *}$ & $1.634^{* *}$ & 0.295 & $0.493^{* *}$ & $0.933^{* *}$ \\
\hline & $(0.167)$ & $(0.173)$ & $(0.242)$ & $(0.163)$ & $(0.177)$ & $(0.312)$ \\
\hline \multirow{2}{*}{$\mathrm{TV}^{\mathrm{c}}$} & 0.061 & 0.320 & 0.396 & -0.463 & 0.009 & 0.018 \\
\hline & $(0.290)$ & $(0.216)$ & $(0.265)$ & $(0.271)$ & $(0.214)$ & $(0.318)$ \\
\hline \multirow{2}{*}{ Radio } & - & $0.363^{*}$ & 0.244 & - & $0.527^{* *}$ & 0.103 \\
\hline & - & $(0.141)$ & (0.199) & - & $(0.151)$ & $(0.259)$ \\
\hline \multirow{2}{*}{ Constante } & $-5.367^{* *}$ & $-2.811^{* *}$ & $-5.382^{* *}$ & $-4.630^{* *}$ & $-4.097^{* *}$ & $-6.598^{* *}$ \\
\hline & $(0.441)$ & $(0.354)$ & $(0.537)$ & $(0.424)$ & $(0.377)$ & $(0.691)$ \\
\hline N & 2,880 & 3,484 & 2,811 & 2,820 & 3,478 & 2,819 \\
\hline Pseudo-R² (McFadden) & 0.438 & 0.222 & 0.291 & 0.399 & 0.252 & 0.234 \\
\hline
\end{tabular}

Elaboración propia en base a estudios del CIS 2736 (2007), 7708 (2008) y 7709 (2009).

Errores Estándar en paréntesis, ** $p<0.01, * p<0.05$

a No disponible en estudio 7708 (2008).

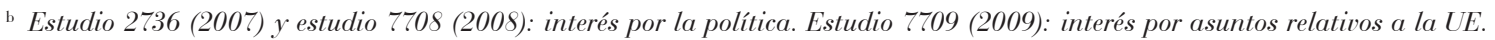

c En estudio 2736 (2007), consumo de noticias sobre política en televisión y radio. 
TABLA 4. ANÁLISIS DE LA BÚSOUEDA Y DE LA RECEPCIÓN DE INFORMACIÓN POLITICA ONLINE (REGRESIONES LOGISTICAS PARA JÓVENES HASTA 30 AÑOS)

\begin{tabular}{|c|c|c|c|c|c|c|}
\hline & $\begin{array}{c}2007 \\
\text { Ha buscado } \\
\text { información } \\
\text { política }\end{array}$ & \begin{tabular}{|c}
2008 \\
Ha buscado \\
información sobre las \\
elecciones generales
\end{tabular} & $\begin{array}{c}2009 \\
\text { Ha buscado } \\
\text { información sobre las } \\
\text { elecciones europeas }\end{array}$ & $\begin{array}{c}2007 \\
\text { Ha recibido un } \\
\text { email con contenido } \\
\text { político }\end{array}$ & $\begin{array}{c}2008 \\
\text { Ha recibido un } \\
\text { email con contenido } \\
\text { político o electoral }\end{array}$ & $\begin{array}{c}2009 \\
\text { Ha recibido un } \\
\text { email con contenido } \\
\text { político o electoral }\end{array}$ \\
\hline \multirow{2}{*}{ Sexo (Mujer) } & -0.261 & $-0.572^{*}$ & -0.082 & 0.255 & 0.015 & 0.208 \\
\hline & $(0.218)$ & $(0.229)$ & $(0.371)$ & $(0.197)$ & $(0.221)$ & $(0.407)$ \\
\hline \multirow{2}{*}{ Edad } & 0.023 & -0.034 & $-0.126^{*}$ & -0.049 & -0.000 & -0.038 \\
\hline & $(0.030)$ & $(0.031)$ & $(0.057)$ & $(0.027)$ & $(0.031)$ & $(0.061)$ \\
\hline \multirow{2}{*}{ Estudios } & $2.638^{* *}$ & $2.233^{* *}$ & $3.871^{* *}$ & $1.914^{* *}$ & $2.098^{* *}$ & 0.590 \\
\hline & $(0.683)$ & $(0.596)$ & (1.195) & $(0.620)$ & $(0.573)$ & $(1.318)$ \\
\hline \multirow{2}{*}{ Ingresos } & 0.082 & -0.955 & 0.964 & $1.471^{*}$ & $1.885^{* *}$ & $2.552^{*}$ \\
\hline & $(0.724)$ & $(0.642)$ & (1.093) & (0.668) & $(0.630)$ & $(1.218)$ \\
\hline \multirow{2}{*}{ Habilidades online ${ }^{a}$} & $3.537^{* *}$ & - & 0.484 & $3.710^{* *}$ & - & 0.293 \\
\hline & $(0.500)$ & - & $(0.381)$ & $(0.463)$ & - & $(0.425)$ \\
\hline \multirow{2}{*}{ Interés por la políticab } & $2.951^{* *}$ & $1.108^{* *}$ & 0.788 & $1.656^{* *}$ & $0.929^{*}$ & $1.544^{*}$ \\
\hline & $(0.423)$ & (0.415) & (0.698) & $(0.375)$ & (0.414) & $(0.781)$ \\
\hline \multirow{2}{*}{ Ideología (derecha) } & -0.517 & 0.127 & 1.620 & 0.473 & -0.569 & -0.275 \\
\hline & $(0.513)$ & $(0.531)$ & $(0.855)$ & $(0.482)$ & $(0.524)$ & (0.999) \\
\hline \multirow{2}{*}{ Partidismo } & 0.413 & -0.015 & $2.858^{* *}$ & 0.228 & $0.550^{*}$ & 1.235 \\
\hline & $(0.220)$ & $(0.245)$ & (0.609) & $(0.201)$ & $(0.246)$ & $(0.679)$ \\
\hline \multirow{2}{*}{ Lectura prensa } & $1.571^{* *}$ & $1.095^{* *}$ & $2.277^{* *}$ & $0.658^{* *}$ & 0.169 & 0.622 \\
\hline & $(0.283)$ & $(0.303)$ & (0.515) & $(0.253)$ & $(0.301)$ & $(0.611)$ \\
\hline \multirow{2}{*}{$\mathrm{TV}^{\mathrm{c}}$} & 0.072 & 0.676 & 0.247 & -0.432 & 0.049 & -0.565 \\
\hline & $(0.429)$ & $(0.378)$ & $(0.583)$ & $(0.387)$ & $(0.340)$ & $(0.599)$ \\
\hline \multirow{2}{*}{ Radio } & - & $0.583^{*}$ & 0.451 & - & $0.594^{*}$ & 0.611 \\
\hline & - & $(0.273)$ & $(0.498)$ & - & $(0.278)$ & $(0.566)$ \\
\hline \multirow{2}{*}{ Constante } & $-6.938^{* *}$ & $-3.271^{* *}$ & $-6.045^{* *}$ & $-4.135^{* *}$ & $-4.835^{* *}$ & $-4.892^{* *}$ \\
\hline & $(0.979)$ & $(0.947)$ & $(1.482)$ & $(0.846)$ & $(0.948)$ & (1.617) \\
\hline $\mathrm{N}$ & 659 & 673 & 465 & 641 & 672 & 467 \\
\hline Pseudo-R² (McFadden) & 0.348 & 0.165 & 0.369 & 0.245 & 0.136 & 0.128 \\
\hline
\end{tabular}

Elaboración propia en base a estudios del CIS 2736 (2007), 7708 (2008) y 7709 (2009).

Errores Estándar en paréntesis, ** $p<0.01, * p<0.05$

a No disponible en estudio 7708 (2008).

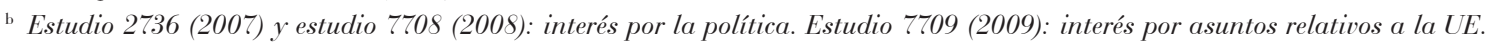

c En estudio 2736 (2007), consumo de noticias sobre política en televisión y radio. 
A pesar de las diferencias en el número de personas que reciben información política por Internet en los tres momentos que se estudian, el tipo de personas que reciben esta información es bastante similar para los tres casos. Tener mayores niveles de estudios y más habilidades en el uso de Internet, interesarse por la política y leer la prensa con mayor frecuencia, aumentan la probabilidad de buscar o recibir información política online en los tres momentos analizados. La edad es también una variable significati$v a$, en la dirección esperada (los más jóvenes están más expuestos), excepto en 2007, para buscar información política. Las mujeres buscan información menos que los hombres en 2007 y 2008, pero no en 2009, ni tampoco parecen recibir menos emails. Los ingresos parecen ser más importantes a la hora de recibir información que a la hora de buscarla. Esto confirma la idea establecida en la literatura de que las personas con posiciones sociales más centrales son objeto de más estímulos movilizadores (Rosenstone y Hansen 1993), aunque no busquen información política online de una manera diferente a quienes tienen menores ingresos.

Simpatizar con un partido tiene el efecto esperado en todos los casos salvo, curiosamente, a la hora de buscar información sobre las elecciones generales: parece que en contextos de mayor movilización la búsqueda de información está menos condicionada por motivaciones previas como la simpatía partidista. Los individuos de izquierda son más proclives a buscar (pero no a recibir) información online, al menos en 2007 y 2008. El uso de la televisión para obtener información política no tiene incidencia sobre la recepción de información online para ninguno de los casos, mientras que la radio tan sólo resulta pertinente en el caso de las elecciones generales.

Si analizamos exclusivamente al grupo de personas hasta 30 años (de los cuales más del 85\% son usuarios de Internet) los resultados son similares con algún pequeño matiz: los recursos cognitivos (educación, habilidades online) parecen ser menos importantes en 2009; la ideología deja de tener un efecto significativo. En cambio el partidismo y el seguimiento de prensa parecen ser más relevantes.

En general, los análisis muestran que tanto los recursos como las motivaciones son variables importantes para explicar la exposición a información política, tal y como esperábamos de la literatura.

\section{LOS EFECTOS DE LA INFORMACIÓN POLÍTICA ONLINE}

Para analizar los efectos del seguimiento o la recepción de información política online nos limitaremos a los contextos electorales de 2008 y 2009 tomando dos variables dependientes: la discusión sobre las elecciones y la participación electoral. Queremos ver si el hecho de buscar información online, o el hecho de recibir un email con contenido político, tienen un efecto sobre la discusión política y el voto, distinto a otros factores con los que están relacionados como el consumo de medios tradicionales, los recursos o las actitudes políticas. Estudios anteriores sobre las consecuencias del uso de Internet sobre la participación política y electoral han encontrado alguna evidencia sobre las influencias positivas del uso de Internet sobre el comportamiento político (Tolbert y McNeal, 2003; Cantijoch, 2009). Estas consecuencias pueden ser el resultado de que Internet provea estímulos adicionales, o de que incluso pueda modificar actitudes políticas. Se ha encontrado que el seguimiento de la campaña online incrementa directamente el grado de conocimiento político, de posicionamiento sobre cuestiones políticas y de participación política no electoral (Xenos y Moy, 2007) y que en cambio el efecto sobre la participación electoral y la discusión depende del nivel de interés por la política (Kenski y Stroud, 2006). De tal forma, las consecuencias de esta información no son necesariamente las mismas para todos los individuos, sino que pueden variar en función de sus características demográficas y sus actitudes hacia la política. La discusión en torno a quienes son más sensibles a las oportunidades que ofrece Internet puede resumirse en un debate que se plantea en las tesis de refuerzo o movilización.

Quienes defienden la perspectiva del refuerzo argumentan que las personas habitualmente implicadas en política son quienes utilizan Internet con objetivos políticos, para informarse, movilizar, o participar (Bimber, 2001; Norris, 2003; Margolis y Resnick, 2000) y que, por tanto, los usos políticos de Internet refuerzan desigualdades políticas y sociales preexistentes. Lusoli (2005) encuentra que la búsqueda de información a través de Internet en Europa está estrechamente relacionada con diferentes indicadores de implicación política en las campañas, y que los factores sociodemográficos tienen un impacto importante, aún cuando se controla por actitudes hacia 
la elección. Di Gennaro y Dutton (2006) también encuentran efectos de refuerzo para el caso británico. Este efecto de refuerzo se ve acentuado porque el acceso a Internet está fuertemente condicionado por los recursos socioeconómicos, y por el hecho de que las habilidades online y la familiaridad con Internet pueden a su vez constituir un recurso necesario para la participación (Krueger, 2002). La edad, la educación o los ingresos son variables que determinan la probabilidad de ser usuario de Internet, pero una vez se centra el análisis en los usuarios de Internet, estos factores no son importantes para explicar su uso con fines políticos (Anduiza, Gallego, Cantijoch, 2010).

La tesis de los usos políticos de Internet como refuerzo a quienes tienen motivaciones políticas previas coincide con el debate sobre la incidencia de los entornos mediáticos en las diferencias de conocimiento político entre grupos de ciudadanos (los llamados knowledge gaps). Internet es un entorno muy rico en información, con gran diversidad de contenidos y posibilidades de elección, que requiere iniciativa por parte de los individuos para acceder a contenidos políticos. Este tipo de medios pueden incrementar las diferencias en conocimiento político entre aquellos con los recursos y motivaciones necesarios para aprovechar la información online, y aquellos sin interés por la política, quienes prefieren dedicar su tiempo online al entretenimiento (Prior, 2005 y 2007; Delli Carpini y Keeter, 2002). Las audiencias de los medios tradicionales como la televisión son más susceptibles de estar expuestas inadvertidamente a información política que pueda incidir, no solo en su conocimiento, sino también en su comportamiento y en su voto. Por el contrario, en un entorno como Internet es más fácil que el individuo seleccione el tipo de contenidos a los que quiere exponerse, eliminando los políticos si no está interesado en ellos.

Una visión más optimista es planteada por quienes argumentan efectos de movilización por cuenta de la información política online, en cuanto sostienen que Internet supone nuevas oportunidades de reequilibrar la distribución del poder político. A nivel individual esto supone que la esfera online es una oportunidad para participar políticamente, la cual resulta atractiva para gente reacia a hacerlo por los canales tradicionales.
Teóricamente, éste sería el caso para los jóvenes, quienes tienen mayores habilidades y facilidad para el uso de Internet y menores niveles de conocimiento e interés por la política. En este caso, de nuevo, es importante distinguir entre acceso a Internet y usos políticos, ya que si bien la edad es un factor significativo a la hora de determinar las probabilidades de ser un internauta, ello no quiere decir que los jóvenes sean los más participativos de aquellas personas con acceso a la red. En nuestro caso probaremos la hipótesis de la movilización prestando especial atención a los jóvenes, que suelen presentar niveles de implicación y participación política inferiores a la media.

Los datos obtenidos para los casos de las elecciones generales de 2008 y las elecciones europeas de 2009 en España confirman la incidencia de la información online sobre la discusión y, en menor medida, sobre el voto. Los resultados se presentan en la tabla 5 . El resultado más contundente de los efectos de la exposición a información política online se da sobre la discusión. Haber buscado información política online y recibido correos con contenidos acerca de la campaña implica un aumento en la frecuencia de discusión sobre política, tanto en 2008 como en 2009. En realidad la recepción de emails y la discusión política pueden considerarse ambas resultados de la densidad de los vínculos sociales de una persona. A la vista de los datos y con las cautelas que impone una única encuesta de sección cruzada, parece que buscar información y recibir emails sobre política generan discusión y que este es uno de los canales de comunicación política relevantes durante la campaña (Norris y Curtice, 2008).

Los efectos de la exposición a información política online sobre el voto se limitan a búsqueda activa de información sobre la decisión de votar o no en las elecciones europeas y no se encuentran efectos en las elecciones generales. Cabe notar que tampoco hay efectos sobre la orientación del voto (resultados no presentados por limitaciones de espacio). Este efecto es mayor para los jóvenes menores de treinta, lo que podria interpretarse como evidencia a favor de la hipótesis de la movilización. De momento no parece que la movilización online a través de correos electrónicos esté teniendo efectos movilizadores sobre los ciudadanos. 
TABLA 5. ANÁLISIS DEL VOTO (REGRESIÓN LOGISTICA) Y DE LA DISCUSIÓN SOBRE POLITICA (REGRESIÓN LINEAL MÚLTIPLE) PARA TODA LA MUESTRA

\begin{tabular}{|c|c|c|c|c|}
\hline & $\begin{array}{l}2008 \\
\text { Voto }\end{array}$ & $\begin{array}{c}2008 \\
\text { Discusión }\end{array}$ & $\begin{array}{l}2009 \\
\text { Voto }\end{array}$ & $\begin{array}{c}2009 \\
\text { Discusión }\end{array}$ \\
\hline \multirow{2}{*}{ Busca información } & -0.113 & $0.042^{* *}$ & $0.465^{*}$ & $0.085^{* *}$ \\
\hline & $(0.242)$ & $(0.013)$ & $(0.210)$ & $(0.015)$ \\
\hline \multirow{2}{*}{ Recibe email } & 0.319 & $0.058^{* *}$ & 0.230 & $0.126^{* *}$ \\
\hline & $(0.279)$ & $(0.014)$ & $(0.258)$ & $(0.019)$ \\
\hline \multirow{2}{*}{ Sexo (Mujer) } & 0.184 & $-0.030^{* *}$ & -0.032 & -0.005 \\
\hline & $(0.129)$ & $(0.009)$ & $(0.092)$ & $(0.008)$ \\
\hline \multirow{2}{*}{ Edad } & $0.061^{* *}$ & $0.010^{* *}$ & 0.024 & $0.006^{* *}$ \\
\hline & $(0.021)$ & $(0.002)$ & $(0.015)$ & $(0.001)$ \\
\hline \multirow{2}{*}{ Edad $^{2}$} & -0.0004 & $-0.0001^{* *}$ & -0.000 & $-0.000^{* *}$ \\
\hline & $(0.000)$ & $(0.000)$ & $(0.000)$ & $(0.000)$ \\
\hline \multirow{2}{*}{ Estudios } & 0.386 & 0.019 & -0.207 & 0.009 \\
\hline & $(0.289)$ & $(0.019)$ & $(0.233)$ & $(0.018)$ \\
\hline \multirow{2}{*}{ Ingresos } & 0.677 & $0.074^{* *}$ & $0.958^{* *}$ & 0.005 \\
\hline & $(0.409)$ & $(0.026)$ & $(0.323)$ & $(0.026)$ \\
\hline \multirow{2}{*}{ Interés en la campaña } & $2.473^{* *}$ & $0.241^{* *}$ & $0.932^{* *}$ & $0.111^{* *}$ \\
\hline & $(0.269)$ & $(0.017)$ & $(0.178)$ & $(0.014)$ \\
\hline \multirow{2}{*}{ Ideología (derecha) } & 0.480 & -0.009 & $0.612^{* *}$ & 0.014 \\
\hline & $(0.347)$ & $(0.021)$ & $(0.226)$ & $(0.018)$ \\
\hline \multirow{2}{*}{ Partidismo } & $1.033^{* *}$ & $0.051^{* *}$ & $0.864^{* *}$ & $0.046^{* *}$ \\
\hline & $(0.138)$ & $(0.009)$ & $(0.091)$ & $(0.008)$ \\
\hline \multirow{2}{*}{ Habilidades online $^{b}$} & - & - & -0.197 & $0.050^{* *}$ \\
\hline & - & - & $(0.214)$ & $(0.018)$ \\
\hline \multirow{2}{*}{ Lectura prensa } & -0.238 & $0.081^{* *}$ & 0.035 & $0.056^{* *}$ \\
\hline & $(0.199)$ & $(0.012)$ & $(0.147)$ & $(0.011)$ \\
\hline \multirow{2}{*}{ TV } & $0.441^{*}$ & $0.059^{* *}$ & $0.783^{* *}$ & $0.111^{* *}$ \\
\hline & $(0.177)$ & $(0.013)$ & $(0.119)$ & $(0.010)$ \\
\hline \multirow{2}{*}{ Radio } & 0.324 & $0.046^{* *}$ & $0.374^{* *}$ & $0.056^{* *}$ \\
\hline & $(0.198)$ & $(0.011)$ & $(0.138)$ & $(0.010)$ \\
\hline \multirow{2}{*}{ Contacto offline } & -0.044 & 0.007 & 0.078 & $0.022^{* *}$ \\
\hline & $(0.128)$ & (0.009) & $(0.098)$ & $(0.008)$ \\
\hline \multirow{2}{*}{ Constante } & $-2.115^{* *}$ & -0.044 & $-2.312^{* *}$ & $-0.066^{*}$ \\
\hline & $(0.540)$ & $(0.039)$ & $(0.391)$ & $(0.032)$ \\
\hline $\mathrm{N}$ & 3,480 & 2,354 & 2,748 & 2,758 \\
\hline Bondad de ajuste ${ }^{c}$ & 0.163 & 0.362 & 0.129 & 0.274 \\
\hline
\end{tabular}

Elaboración propia en base a estudios del CIS 7708 (2008) y 7709 (2009).

Errores Estándar en paréntesis, ** $p<0.01, * p<0.05$.

${ }^{a}$ Estudio 7708 (2008): interés en la campaña. Estudio 7709 (2009): interés por asuntos relativos a la UE.

b No disponible en estudio 7708 (2008).

c Bondad de ajuste: para regresión logística, Pseudo-R (McFadden); Para regresión lineal múltiple, $R^{2}$ ajustada. 
TABLA 6. ANÁLISIS DEL VOTO (REGRESIÓN LOGISTICA) Y DE LA DISCUSIÓN SOBRE POLITICA (REGRESIÓN LINEAL MÚLTIPLE) PARA JÓVENES HASTA 30 AÑOS

\begin{tabular}{|c|c|c|c|c|}
\hline & $\begin{array}{l}2008 \\
\text { Voto }\end{array}$ & Discusión & $\begin{array}{l}2009 \\
\text { Voto }\end{array}$ & Discusión \\
\hline \multirow{2}{*}{ Busca información } & 0.135 & $0.060^{*}$ & $1.103^{* *}$ & $0.121^{* *}$ \\
\hline & $(0.437)$ & $(0.024)$ & $(0.428)$ & $(0.030)$ \\
\hline \multirow{2}{*}{ Recibe email } & 0.324 & 0.039 & 0.334 & $0.128^{* *}$ \\
\hline & $(0.438)$ & $(0.024)$ & $(0.487)$ & $(0.035)$ \\
\hline \multirow{2}{*}{ Sexo (Mujer) } & $0.598^{*}$ & -0.004 & 0.028 & -0.026 \\
\hline & $(0.265)$ & $(0.018)$ & $(0.218)$ & $(0.017)$ \\
\hline \multirow{2}{*}{ Edad } & 0.218 & 0.035 & 0.249 & -0.016 \\
\hline & $(0.513)$ & $(0.037)$ & $(0.401)$ & $(0.032)$ \\
\hline \multirow{2}{*}{$\mathrm{Edad}^{2}$} & -0.004 & -0.001 & -0.006 & 0.000 \\
\hline & $(0.011)$ & $(0.001)$ & $(0.008)$ & $(0.001)$ \\
\hline \multirow{2}{*}{ Estudios } & 0.625 & $-0.097^{*}$ & 0.638 & 0.112 \\
\hline & $(0.668)$ & $(0.045)$ & $(0.750)$ & $(0.058)$ \\
\hline \multirow{2}{*}{ Ingresos } & -0.678 & 0.070 & 1.256 & -0.087 \\
\hline & $(0.785)$ & $(0.052)$ & $(0.734)$ & $(0.056)$ \\
\hline \multirow{2}{*}{ Interés en la campaña } & $4.153^{* *}$ & $0.231^{* *}$ & 0.377 & 0.065 \\
\hline & $(0.638)$ & $(0.038)$ & $(0.434)$ & $(0.034)$ \\
\hline \multirow{2}{*}{ Ideología (derecha) } & 0.235 & $-0.096^{*}$ & -0.347 & -0.028 \\
\hline & $(0.690)$ & $(0.044)$ & $(0.548)$ & $(0.043)$ \\
\hline \multirow{2}{*}{ Partidismo } & $1.383^{* *}$ & $0.039^{*}$ & $1.236^{* *}$ & 0.035 \\
\hline & $(0.298)$ & $(0.020)$ & $(0.216)$ & $(0.018)$ \\
\hline \multirow{2}{*}{ Habilidades online $^{b}$} & - & - & $-1.190^{* *}$ & 0.026 \\
\hline & - & - & $(0.439)$ & $(0.033)$ \\
\hline \multirow{2}{*}{ Lectura prensa } & -0.323 & $0.085^{* *}$ & 0.115 & 0.007 \\
\hline & $(0.421)$ & $(0.026)$ & $(0.362)$ & $(0.029)$ \\
\hline \multirow{2}{*}{ TV } & -0.299 & 0.040 & 0.510 & $0.103^{* *}$ \\
\hline & $(0.388)$ & $(0.027)$ & $(0.298)$ & $(0.024)$ \\
\hline \multirow{2}{*}{ Radio } & 0.976 & $0.057^{*}$ & 0.418 & $0.081^{* *}$ \\
\hline & $(0.512)$ & $(0.026)$ & $(0.371)$ & $(0.028)$ \\
\hline \multirow{2}{*}{ Contacto offline } & 0.178 & 0.035 & 0.356 & 0.011 \\
\hline & $(0.264)$ & $(0.018)$ & $(0.240)$ & (0.019) \\
\hline \multirow{2}{*}{ Constante } & -3.991 & -0.196 & -4.463 & 0.215 \\
\hline & $(6.126)$ & $(0.446)$ & $(4.756)$ & $(0.381)$ \\
\hline $\mathrm{N}$ & 667 & 550 & 450 & 454 \\
\hline Bondad de ajuste $^{c}$ & 0.267 & 0.288 & 0.155 & 0.276 \\
\hline
\end{tabular}

Elaboración propia en base a estudios del CIS 7708 (2008) y 7709 (2009).

Errores Estándar en paréntesis, ** $p<0.01, * p<0.05$.

a Estudio 7708 (2008): interés en la campaña. Estudio 7709 (2009): interés por asuntos relativos a la UE.

b No disponible en estudio 7708 (2008).

c Bondad de ajuste: para regresión logística, Pseudo-R $R^{2}$ (McFadden); Para regresión lineal múltiple, $R^{2}$ ajustada. 


\section{Conclusiones}

Este artículo ha analizado los usos de Internet para el seguimiento de información política online, distinguiendo entre la búsqueda activa y la recepción de emails con contenido político.

El análisis descriptivo sitúa a España entre los paises con menores niveles de exposición a información política online. Por el momento, los medios tradicionales siguen dominando el espacio de las campañas y la información política frente a Internet como alternativa. A pesar de la oferta de los partidos y de la participación de algunos ciudadanos más activos en las campañas a través de blogs y de herramientas que permiten el debate político online, sólo cerca del $20 \%$ de las personas usan Internet como fuente de información política, en el mejor de los casos. España se sitúa aún muy lejos de las cifras de seguimiento de información política online de Estados Unidos. Incluso comparativamente con otros paises europeos, los ciudadanos españoles están muy por debajo de la media en el uso de Internet como fuente de información política.

El análisis explicativo de la exposición a información política online permite ver qué características individuales la facilitan. Los datos nos permiten concluir que hay mayor propensión entre quienes tienen mayores recursos (hombres con mayores niveles de educación, habilidades en el uso de Internet y frecuente lectura de la prensa). También es importante el rol de motivaciones tales como el interés por la política, la campaña electoral 0 , en su caso, los asuntos europeos; la simpatía con un partido o el consumo de información política a través de medios tradicionales. Encontramos además pocas diferencias entre buscar información política y recibir información política o electoral en Internet. En todo caso parece que la exposición a información online, sea a través de búsquedas intencionales o de la recepción de emails, está tan condicionada por recursos y motivaciones como la que procede de otros medios de comunicación tradicionales.

Finalmente, el análisis de las consecuencias de la exposición a información política online se ha centrado en ver de qué manera puede incidir ésta sobre la discusión política y sobre la participación electoral. Éste es sin duda el aspecto más relevante y controvertido de esta cuestión. Nuestros análisis no permiten confirmar ni las teorias más optimistas que esperaban un cambio significativo en la promoción de la implicación política, ni las pesimistas que sugerian que Internet reforzaría las tendencias de desigualdad en el acceso a la información y la implicación política. Los efectos del seguimiento de información política a través de Internet y de la recepción de estímulos movilizadores sobre el voto y la discusión sobre política son moderados, y en realidad no cabe esperar efectos de una magnitud considerable dado que aún nos encontramos ante comportamientos que son extremadamente minoritarios. El seguimiento de información política online y la recepción de emails parecen afectar sobre todo a la discusión política, algo que ya ha sido apuntado en otros países y que nos obliga a contextualizar la comunicación política online dentro del marco más amplio de las campañas electorales.

En la medida en que vayan aumentando los niveles de exposición a información y estímulos online es posible que sus consecuencias se vean incrementadas. Éste es un escenario previsible, dado no solo la creciente difusión de Internet entre la ciudadanía, sino también el mayor uso de Internet por parte de partidos y organizaciones políticas, la multiplicación de webs de información y blogs, y la creciente utilización de entornos web 2.0 como las redes sociales donde se multiplican las oportunidades de exposición.
NOTAS

1 Todos ellos estan disponibles en la web del CIS www.cis.es y se refieren a

Recibido: 15 de septiembre de 2011 Aceptado: 9 de febrero de 2012 muestras representativas de ciudadanos españoles residentes en España.
2 P24. ¿Con qué frecuencia usa Internet para obtener información acerca de la actualidad política? P25. ¿Alguna vez ha consultado la página web de algún partido político o algún candidato? P26. ¿Está usted suscrito a algún bo- 
letín o lista de distribución a través de Internet sobre temas de actualidad política? P27. ¿Alguna vez ha recibido un correo electrónico con alguno de los siguientes contenidos? De apoyo a un candidato o partido/Con alguna crítica sobre un político o un partido político/ Convocando a una manifestación o una sentada o a otros actos de protesta/ Con un manifiesto, una petición electrónica/ Sobre otros temas de actualidad $u$ otro tipo de convocatoria.

3 P23. ¿Ha seguido usted la campaña electoral o se ha informado sobre las elecciones a través de Internet? P23a. ¿Con qué frecuencia? P23b. ¿Podría decirme de las siguientes, en qué tipo de páginas ha entrado para seguir la campaña o informarse de las elecciones? En páginas de medios de comunicación (periódicos, radios, etc.)/ En páginas de partidos o candidatos/En páginas de organizaciones ciudadanas o movimientos cívicos/En blogs y foros de debate/ En otro tipo de páginas.

4 Estudio "La Sociedad en Red 2008" del Observatorio Nacional de las Telecomunicaciones y de la Sociedad de la Información-ONTSI. Como usuario habitual se considera haber utilizado Internet desde cualquier lugar durante el último mes.

5 Evolución de los usos de Internet en España 2009, ONTSI.

6 Según datos del Pew Internet \& American Life Project Poll, la exposición accidental a contenidos de la campaña online es reconocida por el 52\% de los encuestados en 2004 (año de elecciones presidenciales) y el $36 \%$ de los encuestados en 2006.

7 La única información de encuesta sobre el uso de otras herramientas electorales tales como las aplicaciones para seleccionar candidatos y parti- dos (European Election Studies, 2010) muestra que para las Elecciones Europeas de 2009 en España se alcanza un nivel muy bajo (cerca de 2,2\%), por debajo de la media europea de $4,4 \%$. Para los países líderes en uso de estas herramientas (Holanda 21\% o Finlandia $15,4 \%$ ) se ha estimado que su uso en elecciones nacionales pasadas incidió significativamente sobre el nivel de conocimiento politico (Grönlund, 2007).

8 http://www.pewinternet.org/Reports/2008/The-Internet-and-the2008-Election.aspx (consultado 4 de octubre de 2010).

9 En 2008 no hay información sobre si los encuestados son o no usuarios habituales de Internet, por lo que no es posible hacer análisis comparando únicamente usuarios de Internet en los tres momentos. Hemos preferido hacer el análisis con toda la muestra para poder establecer comparaciones entre los tres contextos temporales, pero hay que tener en cuenta que los coeficientes pueden estar en realidad reflejando diferencias en el uso de Internet (y no en la exposición a información política online). Esta limitación se supera parcialmente analizando solo los jóvenes que son todos o casi todos usuarios habituales de Internet (aunque obviamente también presentan otras caracteristicas que los distinguen del conjunto de la muestra). Por ello presentamos los análisis tanto para las muestras completas como para la submuestra de jóvenes de 18 a 30 años.

\section{REFERENCIAS}

Anduiza, Eva; Gallego, Aina y Cantijoch, Marta (2010): "Online Political Participation in Spain: The Impact of
Traditional and Internet Resources", Journal of Information Technology and Politics, vol. 7, n. ${ }^{\circ} 4$, pp. 356-368.

Bimber, Bruce (2001): Information and American Democracy: Technology in the Evolution of Political Power (Communication, Society and Politics), Cambridge University Press.

Bode, Leticia (2010): "Accidentally informed: incidental learning in Facebook", Paper presented for the MPSA, Chicago.

Cantijoch, Marta (2012): "Digital media and offline political participation in Spain", en Anduiza, E., Jensen, M. y Jorba, L. (eds.), Digital Media and Political Engagement Worldwide, Cambridge: Cambridge University Press: 118-137.

Cantijoch, Marta y San Martin, Josep (2008): "Exposure to Political Information in New and Old Media: Which Impact on Political Participation?", Paper presented at the annual meeting of APSA, Boston, Massachusetts.

Delli Carpini, Michael y Scott Keeter (2002): "The internet and an informed citizenry", en Anderson, D. y Cornfield, M. (eds.), The Civic Web, Lanham: Rowman \& Littlefield Publishers, pp. 129153.

Di Gennaro, C. y Dutton, W. H. (2006): "The Internet and the Public: Online and Offline Political participation in the United Kingdom", Parliamentary Affairs, 59 (2): 299-313.

Karlsen, Rune (2007): "Campaign Communication and the Internet: Party Strategy and Voter Use in the 2005 Norwegian Election Campaign", Paper presented at the 4th ECPR General Conference, Pisa, 6-8 September.

Kenski, K. y Stroud, N. J. (2006): "Connections between Internet use and political efficacy, knowledge, and participation", Journal of Broadcasting and Electronic Media, 50 (2), pp. 173-192. 
Krueger, B. S. (2002): "Assessing the Potential of Internet Political Participation in the United States", American Politics Research, 30(5), pp. 476-498.

Lusoli, Wainer (2005): "A second-order medium? The Internet as a source of electoral information in 25 European countries", Information Polity, 10, pp. 247-265.

Margolis, M. y Resnick, D. (2000): Politics as usual: The cyberspace revolution, London, Sage.

Mossberger, K.; Tolbert, C. J. y McNeal, R. S. (2008): Digital Citizenship. Cambridge, MA: The MIT Press.

Norris, P. (2003): "Preaching to the Converted? Pluralism, Participation and Party websites', Party Politics, 9(1): 21-46.

Norris, P. y Curtice, J. (2008): "Getting the Message Out: A Two-Step Model of the Role of the Internet in Campaign Communication Flows During the 2005 British General Election", Journal of Information Technology and Politics, 4: 4, pp. 3-13.

Prior, Markus (2005): "News vs. Entertainment: How Increasing Media Choice Wides Gaps in Political Knowledge and Turnout", American Journal of Political Science, 49(3): 577-92.

Prior, Markus (2007): Post-broadcast democracy: How media choice increases inequality in political involvement and polarizes elections, New York: Cambridge University Press.

Rosenstone, S. J. y Hansen, J. M. (1993): Mobilization, Participation, and Democracy in America, New York: MacMillan.

Tolbert, Caroline J. y McNeal, Ramona S. (2003): "Unraveling the Effects of the
Internet on Political Participation?", Political Research Quarterly, Vol. 56 (2): 175-185.

Vedel T. y Cann Y. M. (2008), "Une communication électorale de rupture? L'internet dans les stratégies des candidats et les pratiques d'information de électeurs", en Perrineau, P. (dir.), Le vote de rupture, Paris, Presses de Sciences Po., pp. 51-75.

Verba, Sidney; Schlozman, Kay y Brady, Henry (1995): Voice and Equality: Civic Voluntarism in American Politics, Cambridge, MA: Harvard University Press.

Xenos, Michael y Moy, Patricia (2007): "Direct and Differential Effects of the Internet on Political and Civic Engagement", Journal of Communication, Vol. 57, pp. 704-718. 
APÉNDICE: CODIFICACIÓN DE LAS VARIABLES

\begin{tabular}{|c|c|c|c|}
\hline Variable & 2007 & 2008 & 2009 \\
\hline $\begin{array}{l}\text { Busca } \\
\text { información }\end{array}$ & \multicolumn{3}{|l|}{$\begin{array}{l}0=\text { No } \\
1=\mathrm{Si}\end{array}$} \\
\hline Recibe email & \multicolumn{3}{|l|}{$\begin{array}{l}0=\mathrm{No} \\
1=\mathrm{Si}\end{array}$} \\
\hline Sexo & \multicolumn{3}{|l|}{$\begin{array}{l}0=\text { Hombre } \\
1=\text { Mujer }\end{array}$} \\
\hline Edad & $\begin{array}{l}\text { En años } \\
18-95\end{array}$ & $\begin{array}{l}\text { En años } \\
18-97\end{array}$ & $\begin{array}{l}\text { En años } \\
18-93\end{array}$ \\
\hline Estudios & \multicolumn{3}{|l|}{$\begin{array}{l}\text { Menos de primarios } \\
\text { Primarios } \\
\text { Secundarios } \\
\text { Superiores } \\
\text { De } 0 \text { a } 1\end{array}$} \\
\hline Ingresos & \multicolumn{3}{|c|}{$\begin{array}{l}10 \text { categorias: de menos de } 300 \text { euros a más de } 6.000 \text { euros } \\
\text { De } 0 \text { a } 1 .\end{array}$} \\
\hline $\begin{array}{l}\text { Habilidades } \\
\text { online }\end{array}$ & $\begin{array}{l}\text { Índice aditivo de } 9 \text { actividades online: comprar, } \\
\text { gestiones bancarias, telefonía ip, descarga ar- } \\
\text { chivos, buscar información, usar correo elec- } \\
\text { trónico, chatear, mantener propio web o blog, } \\
\text { navegar sin objetivo especifico } \\
\text { De } 0 \text { a } 1\end{array}$ & No disponible & $\begin{array}{l}\text { Índice aditivo de } 5 \text { actividades online: } \\
\text { comprar, gestiones bancarias, telefonia } \\
\text { ip, descarga archivos, gestiones con la } \\
\text { administración } \\
\text { De } 0 \text { a } 1\end{array}$ \\
\hline $\begin{array}{l}\text { Interés por la } \\
\text { política }\end{array}$ & $\begin{array}{l}\text { Mucho } \\
\text { Bastante } \\
\text { Poco } \\
\text { Nada } \\
\text { De } 0 \text { a } 1\end{array}$ & $\begin{array}{l}\text { Mucho } \\
\text { Bastante } \\
\text { Poco } \\
\text { Nada } \\
\text { De } 0 \text { a } 1 \\
\text { En modelos tablas } 5 \text { y } 6 \text { : interés en } \\
\text { la campaña. Mismas categorias }\end{array}$ & $\begin{array}{l}\text { Interés por asuntos relativos a la UE. } \\
\text { Mucho } \\
\text { Bastante } \\
\text { Poco } \\
\text { Nada } \\
\text { De } 0 \text { a } 1\end{array}$ \\
\hline Ideología & \multicolumn{3}{|l|}{$\begin{array}{l}\text { De izquierda a derecha } \\
\text { De } 0 \text { a } 1\end{array}$} \\
\hline Partidismo & \multicolumn{3}{|l|}{$\begin{array}{l}\text { Siente simpatía por un partido político } \\
\begin{array}{l}0=\text { No } \\
1=\text { Si }\end{array}\end{array}$} \\
\hline Prensa & $\begin{array}{l}\text { Lee las secciones políticas de los periódicos. } \\
\text { Nunca } \\
\text { Con menor frecuencia } \\
\text { 1-2 dias/semana } \\
\text { 3-4 dias/semana } \\
\text { Todos los dias } \\
\text { De } 0 \text { a } 1\end{array}$ & $\begin{array}{l}\text { Seguimiento de información sobre } \\
\text { campaña en la prensa } \\
\text { Nunca o casi nunca } \\
\text { De vez en cuando } \\
\text { Sólo los fines de semana } \\
2-3 \text { días } \\
4-5 \text { dias } \\
\text { Todos los días } \\
\text { De } 0 \text { a } 1\end{array}$ & $\begin{array}{l}\text { Seguimiento de información sobre } \\
\text { campaña en la prensa } \\
\text { Nunca o casi nunca } \\
\text { De vez en cuando } \\
\text { Sólo los fines de semana } \\
2-3 \text { días } \\
4-5 \text { días } \\
\text { Todos los días } \\
\text { De } 0 \text { a } 1\end{array}$ \\
\hline
\end{tabular}




\section{APÉNDICE: CODIFICACIÓN DE LAS VARIABLES (CONTINUACIÓN)}

\begin{tabular}{|c|c|c|c|}
\hline Variable & 2007 & 2008 & 2009 \\
\hline TV & $\begin{array}{l}\text { Ve o escucha noticias en TV o radio } \\
\text { Nunca } \\
\text { Con menor frecuencia } \\
\text { 1-2 dias/semana } \\
\text { 3-4 dias/semana } \\
\text { Todos los dias } \\
\text { De } 0 \text { a } 1 .\end{array}$ & $\begin{array}{l}\text { Seguimiento de información sobre } \\
\text { campaña en TV } \\
\text { Nunca o casi nunca } \\
\text { De vez en cuando } \\
\text { Sólo los fines de semana } \\
2-3 \text { dias } \\
4-5 \text { días } \\
\text { Todos los dias } \\
\text { De } 0 \text { a } 1\end{array}$ & $\begin{array}{l}\text { Seguimiento de información sobre } \\
\text { campaña en TV } \\
\text { Nunca o casi nunca } \\
\text { De vez en cuando } \\
\text { Sólo los fines de semana } \\
\text { 2-3 dias } \\
\text { 4-5 días } \\
\text { Todos los dias } \\
\text { De } 0 \text { a } 1\end{array}$ \\
\hline Radio & $\begin{array}{l}\text { No disponible } \\
\text { (radio incluida en variable sobre TV) }\end{array}$ & $\begin{array}{l}\text { Seguimiento de información sobre } \\
\text { campaña en la radio } \\
\text { Nunca o casi nunca } \\
\text { De vez en cuando } \\
\text { Sólo los fines de semana } \\
2-3 \text { dias } \\
4-5 \text { días } \\
\text { Todos los dias } \\
\text { De } 0 \text { a } 1\end{array}$ & $\begin{array}{l}\text { Seguimiento de información sobre } \\
\text { campaña en la radio } \\
\text { Nunca o casi nunca } \\
\text { De vez en cuando } \\
\text { Sólo los fines de semana } \\
\text { 2-3 dias } \\
\text { 4-5 días } \\
\text { Todos los dias } \\
\text { De } 0 \text { a } 1\end{array}$ \\
\hline Contacto offline & \multicolumn{3}{|c|}{$\begin{array}{l}\text { Ha sido contactado por un partido político (offline) } \\
0=\text { No } \\
1=\mathrm{Si}\end{array}$} \\
\hline
\end{tabular}

